\title{
Contraccezione ormonale e funzione tiroidea
}

\author{
Francesco Torre ${ }^{1} \cdot$ Aldo E. Calogero $^{1} \cdot$ Rosita A. Condorelli $^{1} \cdot$ Rossella Cannarella $^{1} \cdot$ Antonio Aversa $^{2}$. \\ Sandro La Vignera ${ }^{1}$
}

Accettato: 19 aprile 2020 / Pubblicato online: 17 novembre 2020

(c) The Author(s) 2020

Sommario Le disfunzioni della ghiandola tiroidea rappresentano un gruppo di patologie rilevanti sotto il profilo epidemiologico, specie nel sesso femminile. La terapia ormonale contraccettiva è capace di influenzare la funzione tiroidea modulando i livelli di TBG e SHBG e, sebbene frequentemente prescritta, esistono poche evidenze riassuntive circa i limiti prescrittivi nelle donne con disfunzione tiroidea. Lo scopo di questo articolo è quello di rivedere gli effetti del trattamento con levo-tiroxina (LT4) nelle donne in terapia ormonale contraccettiva e come quest' ultima influenzi la funzione tiroidea. La componente estrogenica è responsabile dell' aumento di TBG, SHBG e dei fattori di coagulazione. Per contro, la componente progestinica, attraverso l'azione anti-androgenica, è utile per mantenere basso il rischio tromboembolico e cardiovascolare. Ciò assume particolare rilevanza nelle donne affette da ipotiroidismo subclinico e in quelle trattate con LT4. Infatti, l'ipotiroidismo subclinico è associato a un aumentato volume piastrinico medio, il quale aumenta, a sua volta, il rischio cardiovascolare a causa dell'iperattività piastrinica dovuta alla incompleta maturazione megacariocitica.

Proposto da Antonio Aversa.

Materiale elettronico supplementare La versione elettronica di questo articolo (https://doi.org/10.1007/s40619-020-00797-x) contiene materiale supplementare, disponibile per gli utenti autorizzati.

$\varangle$ S. La Vignera

sandrolavignera@unict.it

1 Dipartimento di Medicina Clinica e Sperimentale dell'Università di Catania, Catania, Italia

2 Dipartimento di Medicina Sperimentale e Clinica dell'Università "Magna Graecia", Catanzaro, Italia
Parole chiave Contraccezione ormonale .

Estroprogestinici · Terapia ormonale sostitutiva · Funzione tiroidea $\cdot \mathrm{SHBG} \cdot$ Ipotiroidismo subclinico

\section{Introduzione}

La sintesi e la secrezione tiroidea dei due ormoni iodati, tiroxina (T4) e triiodotironina (T3) sono controllate dall'ormone tireotropo (TSH) ipofisario, a sua volta controllato dal fattore di rilascio ipotalamico TRH. La massima parte dell'ormone biologicamente attivo, la T3, è prodotta nei tessuti periferici da desiodazione della T4. Gli ormoni tiroidei circolano legati specificamente a una globulina (Thyroxinbinding globulin, TBG) e con minore specificità alla transiretina e all'albumina. Oltre il 99,8\% degli ormoni circolanti è legato a queste proteine, per il $70 \%$ alla TBG e fino al $15 \%$ alla transiretina, con un'affinità 10 volte superiore per la T4 rispetto alla T3. L'elevato peso molecolare del complesso ormone-proteina ne impedisce la filtrazione glomerulare renale, così costituendo una scorta di ormone tiroideo nel compartimento plasmatico. Sono le quote libere di T4 (FT4) e di T3 (FT3) a esercitare il feedback negativo sul rilascio di TSH e TRH in modo da garantire il fisiologico equilibrio metabolico [1]. L'ipertiroidismo e l'ipotiroidismo, esercitano opposte e specifiche ripercussioni metaboliche e sono in grado di influenzare negativamente la funzione gonadica in entrambi i sessi [1].

Le quote libere circolanti di ormone tiroideo sono in equilibrio con quelle legate alle proteine di trasporto. La terapia ormonale contraccettiva è in grado di modificare le concentrazioni delle proteine leganti gli ormoni tiroidei, producendone un aumento o una riduzione, a seconda del tipo di contraccettivo utilizzato.

Lo scopo di questa Rassegna è duplice: se, da un lato, si propone di comprendere se e come la contraccezio- 
ne ormonale possa influenzare l'economia tiroidea, dall'altro cerca di chiarire se l'assunzione di levo-tiroxina (LT4) possa modificare l'efficacia o la sicurezza della terapia contraccettiva.

\section{Contraccettivi orali e diagnostica funzionale tiroidea}

\section{Effetti degli estrogeni}

La causa più comune di un incremento delle concentrazioni sieriche di TBG è l'aumento della produzione di estrogeni o la loro somministrazione, sia come componenti della pillola contraccettiva che della terapia ormonale sostitutiva. In particolare, gli estrogeni naturali sono in grado di aumentare il grado di sialilazione della TBG condizionante, a sua volta, una riduzione della clearance renale e un aumento delle concentrazioni sieriche [2]. La dose più comunemente utilizzata di etinil-estradiolo è 20-35 $\mu \mathrm{g} / \mathrm{die}$, mentre degli estrogeni coniugati è $0,625 \mathrm{mg} / \mathrm{die}$. Entrambe le formulazioni sono in grado di determinare un aumento della TBG del $30-50 \%$ e delle concentrazioni di T4 del 20-35\%. L'aumento comincia a partire dalla seconda settimana di somministrazione e raggiunge il plateau in circa 4-8 settimane. Nelle donne affette da ipotiroidismo in terapia sostitutiva con LT4, è solitamente richiesto un aumento del dosaggio di LT4 fino a $45 \%$ in presenza di un aumento endogeno (gravidanza) o esogeno (terapia ormonale) dei livelli di estrogeni circolanti per mantenere i livelli di TSH entro i limiti di normalità [3]. Questo riflette l'aumento delle concentrazioni di TBG (con conseguente riduzione delle quote di FT3 e FT4) estrogeno-dipendente.

Gli estrogeni orali, comunemente usati come terapia ormonale sostitutiva più che a scopo contraccettivo, hanno un effetto di primo passaggio epatico. Inoltre, la somministrazione transdermica degli estrogeni non aumenta i livelli i TBG o T4, sebbene le concentrazioni ematiche raggiunte con la terapia transdermica e orale siano sovrapponibili [4].

\section{Effetti del progesterone e dei progestinici}

Le evidenze disponibili in letteratura circa l'effetto del solo progesterone (o progestinico) non associato a terapia estrogenica sulla funzione tiroidea sono scarse. Uno studio randomizzato, placebo-controllato, della durata di 12 settimane, ha riportato che il progesterone micronizzato, somministrato alla dose orale di $300 \mathrm{mg}$, riduceva i livelli di TSH, aumentava i livelli di FT4 e non aveva effetto sui livelli di FT3 rispetto al placebo [5]. Inoltre, il trattamento con medrossiprogesterone-acetato è risultato capace di aumentare significativamente i livelli di FT4 più di quanto osservato nelle pazienti trattate con dispositivo intrauterino (IUD) in rame [6]. Gli effetti di ciascun progestinico sull'economia tiroidea sono diversi e sono schematizzati nella Tabella 1.

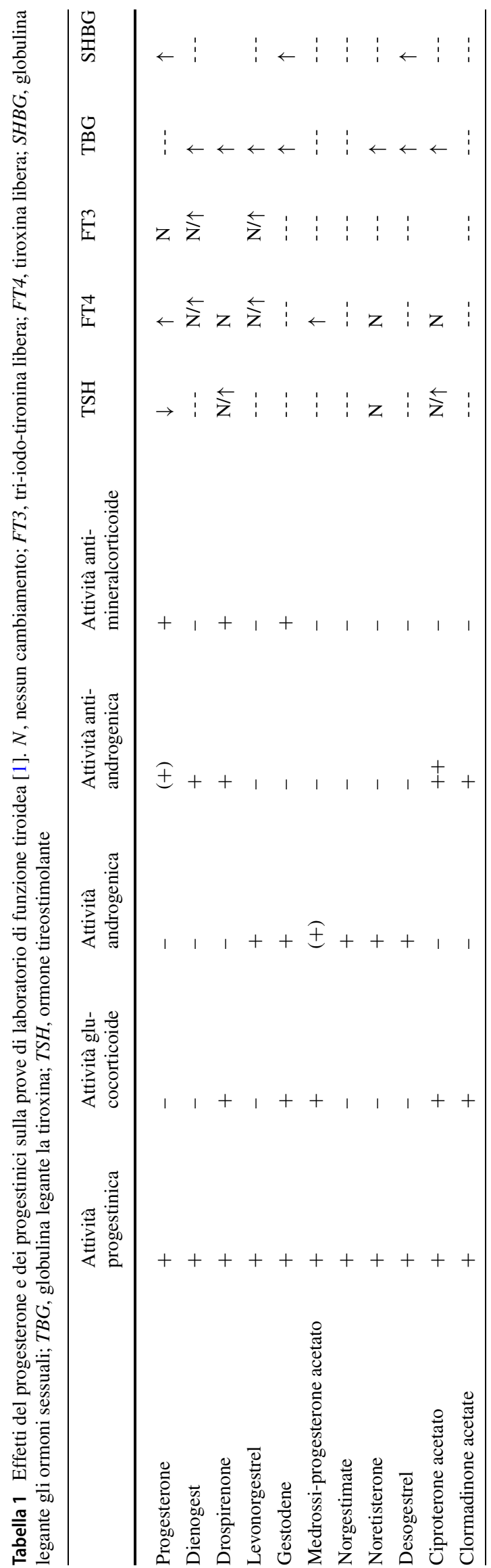


La maggior parte delle evidenze disponibili si riferisce agli effetti della terapia combinata (estro-progestinica) sulla funzione tiroidea. Il dienogest (DNG) presenta un'azione anti-androgenica maggiore rispetto al levonorgestrel (LNG). Il profilo estrogenico del DNG è inoltre leggermente maggiore rispetto a quello del LNG, mentre l'azione anti-estrogenica è scarsa. L'utilizzo combinato degli estrogeni e progestinici dovrebbe tenere in considerazione dell'effetto pleiotropico e sinergico di entrambi questi ormoni. Uno studio randomizzato, doppio-cieco, controllato, ha valutato l'effetto di quattro diversi contraccettivi sulla funzione tiroidea, in particolare il primo contenente $30 \mu \mathrm{g}$ di etinil-estradiolo e $2 \mathrm{mg}$ di DNG, il secondo $20 \mu \mathrm{g}$ di etinilestradiolo e $2 \mathrm{mg}$ di DNG, il terzo $10 \mu \mathrm{g}$ di etinil-estradiolo, $2 \mathrm{mg}$ di estradiolo valerato e $2 \mathrm{mg}$ di DNG e il quarto contenete $20 \mu \mathrm{g}$ di etinil-estradiolo e $100 \mu \mathrm{g}$ di LNG. Lo studio ha riportato un aumento significativo delle concentrazioni di T4 e T3 del 20-40\% in tutti i trattamenti, mentre il TSH è aumentato significativamente solo nelle donne trattate con la terza formulazione [7]. Tale aumento può essere attribuito agli effetti delle terapie contraccettive contenenti DNG o LNG sulle globuline leganti. Lo studio delle quote libere ha riportato cambiamenti minimi e non significativi. Pertanto, queste evidenze indicano chiaramente che i contraccettivi contenenti DNG o LNG hanno solo minimi effetti sulla funzione tiroidea [7].

\section{Influenza della terapia con levotiroxina $(L-T 4)$ sull'efficacia e sicurezza della contraccezione ormonale}

La questione relativa alla possibile influenza che la terapia con LT4 può avere sull'efficacia e sicurezza della terapia contraccettiva deriva da uno studio secondo il quale gli ormoni tiroidei faciliterebbero l'espressione del recettore per gli estrogeni [8]. Per contro, la tiroidectomia totale induce una riduzione dell'espressione dei recettori per gli estrogeni fino al $70 \%$ nel modello murino. La somministrazione di T3 alla dose di $1 \mu \mathrm{g} /$ die normalizzava l'espressione sia del trascritto che della proteina [8]. Tali limitate evidenze sperimentali richiedono comunque studi nella specie umana.

\section{Ripercussioni sul rischio cardiovascolare}

\section{Contraccettivi orali e rischio tromboembolico}

La terapia contraccettiva è associata a un aumento del rischio trombotico di 3-8 volte superiore [9]. Quest'ultimo dipende dalla dose dell'estrogeno e dalla molecola ad azione progestinica utilizzata. Alcune evidenze mostrano come l'effetto della componente progestinica sui livelli di TBG dipenda dall'azione trombogenica intrinseca della molecola.
Se da un lato, infatti, il ciproterone acetato o il drospirenone, aventi un maggiore potenziale trombogenico, provocano un aumento dei livelli di TBG, progestinici con profilo trombogenico migliore (es. LNG) ne determinano una diminuzione [9]. Secondo la Società Americana della Medicina della Riproduzione (ASRM), l'incidenza di tromboembolismo venoso è pari a 16-22 casi su 10.000 donne l'anno [10]. Diversi dati epidemiologici confermano un rischio trombotico più alto nelle donne che utilizzano terapia estrogenica. Tale rischio è ancora maggiore quando a questa viene aggiunto il progesterone [11]. Una recente meta-analisi ha riportato un aumento del rischio trombotico nelle donne in terapia orale con estrogeni, ma non in quelle trattate per via transdermica [12]. Questo suggerisce come la formulazione transdermica possa essere preferibile a quella orale per garantire un minore rischio trombotico.

Le evidenze riportate giustificano, quindi, l'importanza della valutazione del rischio tromboembolico preliminarmente alla prescrizione della terapia contraccettiva.

\section{Volume piastrinico medio e trombosi}

Un aspetto che merita di essere guardato con attenzione nella valutazione del rischio trombotico preliminarmente alla prescrizione della terapia ormonale contraccettiva è rappresentato dal volume piastrinico medio. Le piastrine possono variare in dimensioni. Un aumentato volume piastrinico ha un maggiore potenziale pro-trombotico ed è associato a una più alta resistenza al trattamento con Cardioaspirina ${ }^{\circledR}$ o clopidogrel. Studi recenti hanno riscontrato che l'aumento del volume piastrinico medio è associato a un maggior rischio di trombosi venosa profonda, infarto del miocardio e riveste un ruolo prognostico nei pazienti con malattia cardiovascolare. In particolare, una meta-analisi condotta su 18 studi ha dimostrato che i pazienti con trombosi venosa profonda mostrano valori di volume piastrinico medio più alti rispetto al gruppo controllo [13]. Un aumento del volume piastrinico medio è stato osservato anche nelle malattie dell' apparato cardiovascolare, respiratorio, nell'insufficienza renale cronica, diabete mellito, tumori, malattie intestinali, reumatologiche.

Gli ormoni sessuali possono influenzare la funzione piastrinica. Alti livelli di testosterone aumentano l'espressione del recettore piastrinico per il trombossano A2, favorendo processi di aggregazione. Ciò potrebbe contribuire al maggiore rischio trombotico degli androgeni [14]. La somministrazione di progesterone è capace di aumentare l'affinità di legame della globulina di trasporto degli steroidi sessuali (SHBG), riducendo i livelli di testosterone libero e, quindi, il rischio trombotico (Tabella 1) [15]. Inoltre, la conta piastrinica media diminuisce nel periodo post-menopausale nelle donne che non assumono terapia ormonale contraccettiva, in assenza di significative variazioni del volume piastrinico 
medio. Questo potrebbe far ipotizzare un ruolo per gli estrogeni nel modulare la conta piastrinica media. In aggiunta, nel periodo post-menopausale è stato osservato un aumento della percentuale di piastrine reticolate, queste ultime caratterizzate da una più alta capacità trombotica [16]. Ciononostante, le evidenze disponibili non hanno mostrato una definita interferenza della terapia ormonale contraccettiva nei confronti della funzione piastrinica [17].

Per contro, gli ormoni tiroidei possono influenzare la funzione piastrinica. In particolare, l'ipertiroidismo conclamato è associato a un aumento del rischio trombotico, in quanto un aumento dei livelli di FT4 causa uno stato di ipercoagulabilità. Più in dettaglio, il recettore per la T4 è capace di interagire con l'integrina piastrinica $\alpha \mathrm{v} \beta 3$, iniziando il meccanismo di aggregazione a cascata. Gli ormoni tiroidei sono inoltre in grado di stimolare l'interazione tra le piastrine e l'endotelio, favorendo processi trombotici [18]. Le evidenze della letteratura attribuiscono un ruolo agli ormoni tiroidei nell'influenzare il valore del volume piastrinico medio: mentre gli studi di associazione tra ipertiroidismo conclamato e volume piastrinico medio mostrano risultati contrastanti, l'ipotiroidismo è più chiaramente associato a un aumento del volume piastrinico medio.

\section{Disfunzione tiroidea: influenze sulla funzione arteriosa e sul profilo lipidico}

L'ipotiroidismo si associa a effetti dannosi sul sistema cardiovascolare. Alcuni studi dimostrano, infatti, maggiori valori di pressione sistolica nei pazienti affetti da ipotiroidismo subclinico rispetto ai pazienti con normale funzione tiroidea. L'ipotiroidismo subclinico sembra inoltre correlare a un alterato profilo lipidico, seppure le evidenze su questo punto siano a tratti poco chiare. In particolare, alcuni dati mostrano l'associazione tra ipotiroidismo subclinico e ipertrigliceridemia. Questa evidenza non è però confermata da altri studi e, inoltre, il meccanismo fisiopatologico attraverso cui questa associazione sussisterebbe non è noto. Altre evidenze hanno riscontrato come la terapia con LT4 sia in grado di ridurre i livelli di colesterolo totale e LDL, ma non dei trigliceridi. Comunque, una meta-analisi ha riportato un maggior livello di colesterolo totale, LDL e trigliceridi nei pazienti affetti da ipotiroidismo subclinico rispetto ai pazienti con normale funzione tiroidea [19].

La contraddittorietà dei dati potrebbe essere attribuibile all'eterogeneità delle popolazioni studiate o a bias intrinseci agli studi. L'ipotiroidismo subclinico potrebbe causare nel lungo termine alterazioni del profilo lipidico e del volume piastrinico medio, favorendo l'insorgenza di eventi cardiovascolari. L'aumento del TSH può causare, infatti, un incremento del colesterolo LDL, aterogeno, dei livelli di stress ossidativo e della pressione diastolica, promuovendo la disfunzione endoteliale [20]. Queste evidenze sotto- lineano l'importanza della valutazione della funzione tiroidea preliminarmente alla prescrizione della terapia ormonale contraccettiva, dato l'impatto che essa stessa ha sul rischio trombotico e cardiovascolare.

\section{Conclusioni}

Dalle evidenze riportate si può concludere che la terapia ormonale contraccettiva causa cambiamenti dei livelli di TSH e delle quote libere degli ormoni tiroidei. L'aumento di T3 e T4 è attribuibile alle modifiche della TBG indotte dalla terapia contraccettiva. L'incremento dei livelli di FT3 e FT4, la bassa clearance renale della TBG e il conseguente aumento della sintesi degli ormoni tiroidei devono essere valutate con attenzione, specialmente nei pazienti con ipertiroidismo conclamato o con ipotiroidismo subclinico. Pertanto, la prescrizione della terapia ormonale contraccettiva dovrebbe associarsi a un monitoraggio della funzione tiroidea. L'ipotiroidismo richiede, inoltre, che venga prestata attenzione anche al rischio cardiovascolare, potendosi associare ad alterazioni del profilo lipidico, pressorio e alterazioni coagulative, condizioni favorenti la disfunzione endoteliale e, quindi, associate a un maggiore rischio trombotico e cardiovascolare [1].

Funding Note Open access funding provided by Università degli Studi di Catania within the CRUI-CARE Agreement.

Conflitto di interesse Gli autori Francesco Torre, Aldo E. Calogero, Rosita A. Condorelli, Rossella Cannarella, Antonio Aversa e Sandro La Vignera dichiarano di non avere conflitti di interesse.

Consenso informato Lo studio presentato in questo articolo non ha richiesto sperimentazione umana.

Studi sugli animali Gli autori di questo articolo non hanno eseguito studi sugli animali.

Nota della casa editrice Springer Nature rimane neutrale in riguardo alle rivendicazioni giurisdizionali nelle mappe pubblicate e nelle affiliazioni istituzionali.

Open Access This article is licensed under a Creative Commons Attribution 4.0 International License, which permits use, sharing, adaptation, distribution and reproduction in any medium or format, as long as you give appropriate credit to the original author(s) and the source, provide a link to the Creative Commons licence, and indicate if changes were made. The images or other third party material in this article are included in the article's Creative Commons licence, unless indicated otherwise in a credit line to the material. If material is not included in the article's Creative Commons licence and your intended use is not permitted by statutory regulation or exceeds the permitted use, you will need to obtain permission directly from the copyright holder. To view a copy of this licence, visit http://creativecommons.org/licenses/by/4.0/. 


\section{Bibliografia}

1. Torre F, Calogero AE, Condorelli RA et al (2020) Effects of oral contraceptives on thyroid function and vice versa. J Endocrinol Invest 43(9): 1181-1188

2. Bartalena L, Robbins J (1992) Variations in thyroid hormone transport proteins and their clinical implications. Thyroid $2: 237-245$

3. Mandel SJ, Larsen PR, Seely EW et al (1990) Increased need for thyroxine during pregnancy in women with primary hypothyroidism. N Engl J Med 323(2):91-96

4. Dhopesh VP, Burke WM, Maany I et al (1991) Effect of cocaine on thyroid functions. Am J Drug Alcohol Abuse 17:423-427

5. Sathi P, Kalyan S, Hitchcock CL et al (2013) Progesterone therapy increases free thyroxine levels-data from a randomized placebo-controlled 12-week hot flush trial. Clin Endocrinol (Oxf) 79(2):282-287

6. Quintino-Moro A, Zantut-Wittmann DE, Silva Dos Santos PN et al (2019) Thyroid function during the first year of use of the injectable contraceptive depot medroxyprogesterone acetate. Eur J Contracept Reprod Health Care 24(2):102-108

7. Wiegratz I, Kutschera E, Lee JH et al (2003) Effect of four oral contraceptives on thyroid hormones, adrenal and blood pressure parameters. Contraception 67(5):361-366

8. Vasudevan N, Ogawa S, Pfaff D (2002) Estrogen and thyroid hormone receptor interactions: physiological flexibility by molecular specificity. Physiol Rev 82(4):923-944

9. Raps M, Curvers J, Helmerhorst FM et al (2014) Thyroid function, activated protein $\mathrm{C}$ resistance and the risk of venous thrombosis in users of hormonal contraceptives. Thromb Res 133(4):640-644

10. Practice Committee of the American Society for Reproductive Medicine (2006) Estrogen and progestogen therapy in postmenopausal women. Fertil Steril 86(5):S75-S88
11. Curb JD, Prentice RL, Bray PF et al (2006) Venous thrombosis and conjugated equine estrogen in women without a uterus. Arch Intern Med 166:772-780

12. Scarabin PY (2018) Progestogens and venous thromboembolism in menopausal women: an updated oral versus transdermal estrogen meta-analysis. Climacteric 21(4):341-345

13. Kovács S, Csiki Z, Zsóri KS et al (2019) Characteristics of platelet count and size and diagnostic accuracy of mean platelet volume in patients with venous thromboembolism. A systematic review and meta-analysis. Platelets 30(2):139-147

14. Ajayi AA, Mathur R, Halushka PV (1995) Testosterone increases human platelet thromboxane $\mathrm{A} 2$ receptor density and aggregation responses. Circulation 91(11):2742-2747

15. Dalton ME (1984) The effect of progesterone administration on sex hormone binding globulin binding capacity in women with severe premenstrual syndrome. J Steroid Biochem 20(1):437-439

16. Butkiewicz AM, Kemona $\mathrm{H}$, Dymicka-Piekarska $\mathrm{V}$ et al (2006) Does menopause affect thrombocytopoiesis and platelet activation? Przegl Lek 63(12):1291-1293

17. Saleh AA, Ginsburg KA, Duchon TA et al (1995) Hormonal contraception and platelet function. Thromb Res 78(4):363-367

18. Davis PJ, Mousa SA, Schechter GP (2018) New interfaces of thyroid hormone actions with blood coagulation and thrombosis. Clin Appl Thromb Hemost 24(7):1014-1019

19. Liu XL, He S, Zhang SF et al (2014) Alteration of lipid profile in subclinical hypothyroidism: a metaanalysis. Med Sci Monit 20:1432-1441

20. La Vignera S, Condorelli R, Vicari E et al (2012) Endothelial dysfunction and subclinical hypothyroidism: a brief review. J Endocrinol Invest 35(1):96-103 